\title{
High rate epitaxial lift-off of InGaP films from GaAs substrates
}

\author{
J. J. Schermer,a) G. J. Bauhuis, P. Mulder, W. J. Meulemeesters, E. Haverkamp, \\ M. M. A. J. Voncken, and P. K. Larsen \\ Research Institute for Materials, University of Nijmegen, 6525 ED Nijmegen, The Netherlands
}

(Received 7 December 1999; accepted for publication 10 February 2000)

Centimeter sized, crack-free single crystal InGaP films of $1 \mu \mathrm{m}$ thickness were released from GaAs substrates by a weight-induced epitaxial lift-off process. At room temperature, the lateral etch rate of the process as a function of the applied $\mathrm{Al}_{0.85} \mathrm{Ga}_{0.15} \mathrm{As}$ release layer thickness was found to have a maximum of $3 \mathrm{~mm} / \mathrm{h}$ at $3 \mathrm{~nm}$. Using 5-nm-thick AlAs release layers, the etch rate increased exponentially with temperature up to $11.2 \mathrm{~mm} / \mathrm{h}$ at $80^{\circ} \mathrm{C}$. Correlation of the experimental data with the established theoretical description of the process indicate that the model is qualitatively correct but fails to predict the etch rates quantitatively by orders of magnitude. (C) 2000 American Institute of Physics. [S0003-6951(00)01715-0]

The epitaxial lift-off (ELO) technique in which a III/V device structure is separated from its GaAs substrate using selective wet etching of a thin $\mathrm{Al}_{x} \mathrm{Ga}_{1-x} \mathrm{As}(x>0.6)$ release layer, ${ }^{1}$ has a large potential for optoelectronic applications. The technique results in a significant cost reduction of III/V devices since the GaAs substrate can be reused ${ }^{2,3}$ after the lift-off process. This is of particular importance for intrinsically large area, thus expensive devices like high efficiency III/V solar cells ${ }^{2-4}$ and the integration of high quality optoelectronic III/V structures on silicon large scale integrated circuits. ${ }^{5,6}$

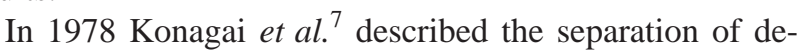
vices from a GaAs substrate using the extreme selectivity of $\mathrm{HF}$ acid for $\mathrm{AlGaAs}$ with a high $\mathrm{Al}$ fraction. A wax layer was applied to support the circa $30-\mu \mathrm{m}$-thick fragile films during the process. Yablonovitch et al. ${ }^{1}$ noted that the tension induced by the wax caused thinner films, of micrometer thickness, to curl up with a radius of curvature $R$ as they became undercut. This was concluded to be beneficial for removal of the etch products, leading to an increased lateral etch rate $V_{e}$ of the AlAs release layer. By assuming that three moles of $\mathrm{H}_{2}$ gas are produced for every mole of AlAs and that the ability to diffuse away the dissolved hydrogen, which has a low solubility limits the etch rate during the ELO process, they derived an expression for $V_{e}$ :

$$
V_{e}=\frac{1}{\pi \sqrt{R h / 2}} \frac{D n}{3 N}
$$

where $n$ and $N$ are the molar concentrations of dissolved $\mathrm{H}_{2}$ and AlAs, respectively, $D$ the diffusion coefficient of $\mathrm{H}_{2}$ in the solution and $h$ the thickness of the release layer which generally is $10 \mathrm{~nm} .{ }^{2-6}$ Based on Eq. (1) Maeda et al. ${ }^{5}$ argued that $V_{e}$ increases exponentially with temperature like $D$ :

$$
D=D_{0} e^{-E_{a} / k T},
$$

where $D_{0}$ is the diffusion coefficient at infinite temperature, $E_{a}$ is the activation energy, and $k$ is the Boltzmann constant. Both relations (1) and (2) hold irrespectively of the III/V

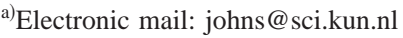

device that is to be separated from the substrate, provided that the gap between them is opened with a certain curvature $R$ and out diffusion of $\mathrm{H}_{2}$ is limiting the etch rate.

In the present work the lateral etch rate of the release layers as a function of their thickness and the temperature applied during the ELO process is determined experimentally and compared with theory. For this purpose the application of the ELO method for InGaP layers is examined instead of the generally used $\mathrm{Al}_{x} \mathrm{Ga}_{1-x} \mathrm{As}(x \leqslant 0.4)$ based devices. ${ }^{1-8}$ Simultaneous etching of InGaP and GaAs layers in a $10 \% \mathrm{HF}$ solution in water performed in our laboratory showed that InGaP etches about two times faster than GaAs. This difference between $\mathrm{InGaP}$ and GaAs is insignificant since the etch rate for GaAs is about $10^{7}$ smaller than for $\mathrm{Al}_{x} \mathrm{Ga}_{1-x} \mathrm{As}^{9}{ }^{9}$ Therefore it is possible to separate thin-film InGaP layers from the GaAs substrate by the use of an $\mathrm{Al}(\mathrm{Ga})$ As release layer. The thickness of the released $\mathrm{InGaP}$ layers was $1 \mu \mathrm{m}$ in all experiments and either $\mathrm{Al}_{0.85} \mathrm{Ga}_{0.15} \mathrm{As}$ or AlAs release layers were used. These III/V structures were grown on 2-in.-diam (100) GaAs wafers by low pressure metal organic vapor phase epitaxy.

A weight-induced ELO (WI-ELO) procedure able to free large area thin films has been developed in our laboratory. The procedure which has been described in detail in previous work $^{2,3}$ utilizes a copper support layer and a HF resistant plastic foil mounted with black wax on top of the $\mathrm{InGaP}$ structures. The samples are connected to supports at the lid of a HF resistant container and a weight is fitted in the excess foil at one side of the sample (see Fig. 1). One droplet of $10 \% \mathrm{HF}$ solution, positioned on the plastic foil against the edge of the sample at the side of the weight, is sufficient to perform the ELO process under conditions usually applied. Elevated temperatures in the range between 50 and $80^{\circ} \mathrm{C}$ at the position of the samples were obtained by the application of a HF resistant rubber heater element at the bottom of the container. Using this approach, crack-free InGaP films could be obtained with a size up to $34 \times 32 \mathrm{~mm}^{2}$, which is the entire 2 in. wafer minus cleavage losses. For the other experiments the wafers were cleft in pieces of $15 \times 15 \mathrm{~mm}^{2}$ and three identical samples were placed in the container simultaneously to determine the reproducibility of the process. $V_{e}$ is 


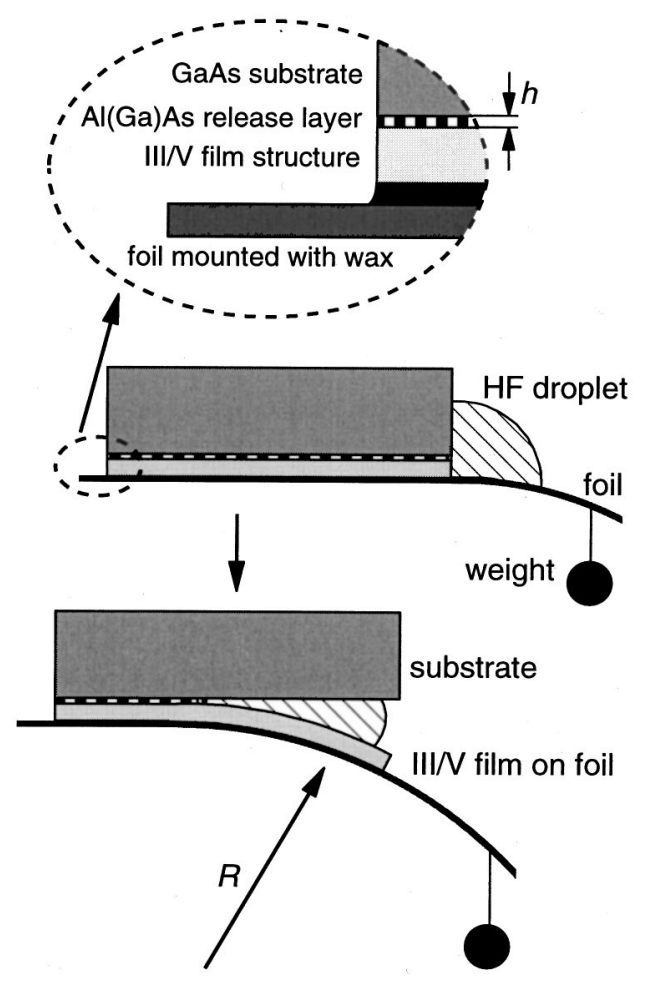

FIG. 1. Schematic cross section of the sample configuration during the WI-ELO process. The relative size of the different parts is greatly out of proportion.

given by averaging the lateral etch rates over these samples while a spread in etch rate as large as $30 \%$ between the fastest and slowest sample released was observed under all conditions examined.

$V_{e}$ as a function of the applied $\mathrm{Al}_{0.85} \mathrm{Ga}_{0.15}$ As release layer thickness is shown in Fig. 2. In accordance with Eq. (1), the results reveal a linear relation between $h^{-1 / 2}$ and $V_{e}$ for $h$ in the range from 3 to $40 \mathrm{~nm}$. Samples with thinner release layers did not show any under etching while most samples with $h>40 \mathrm{~nm}$ revealed only partial under etching. The etch process of the latter came to a hold because after several days in the WI-ELO container the HF droplet on these samples was found to be evaporated.

Based on these results a $5 \mathrm{~nm}$ release layer thickness was chosen as a standard for the experiments at elevated tempera-

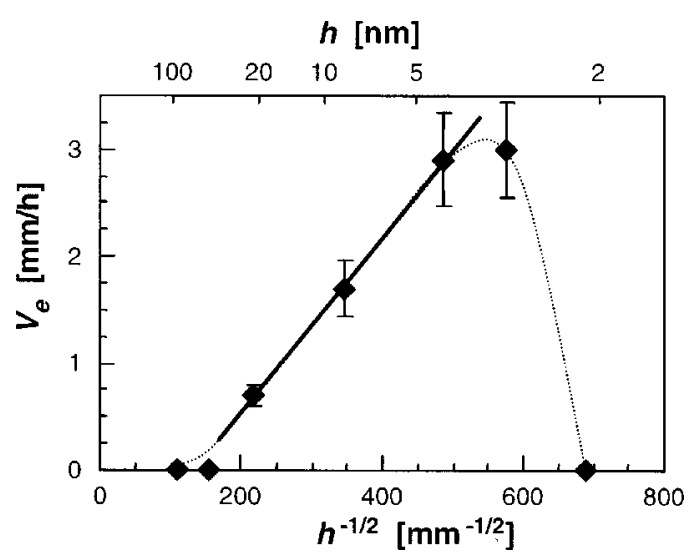

FIG. 2. Lateral etch rate of the $\mathrm{Al}_{0.85} \mathrm{Ga}_{0.15}$ As release layer in $10 \% \mathrm{HF}$ solution as a function of layer thickness. The dashed line is to guide the eye and the solid line indicates the best linear fit through the data obtained between 4 and $40 \mathrm{~nm}$.

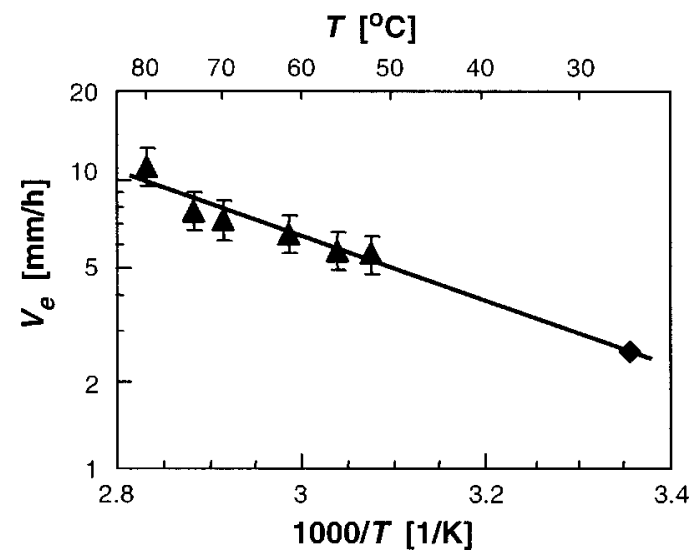

FIG. 3. Lateral etch rate of 5-nm-thick AlAs release layers in a $10 \% \mathrm{HF}$ solution as a function of temperature. The solid line is the best linear fit through the data points and the diamond marker represents the etch rate for a $5 \mathrm{~nm}$ release layer at room temperature as obtained from Fig. 2 .

tures and the WI-ELO container was modified to facilitate access with a pipette in order to apply a new droplet of HF whenever the previous would be nearly evaporated. In practice it turned out difficult to refresh the HF droplet during the experiments and in several occasions the droplet adhered not only to the front edge but also to the side rims of the sample. Since such a sample is simultaneously etched from more than one side, this results in a significant faster release of the InGaP layer from its substrate. On one such occasion, a 15 $\times 15 \mathrm{~mm}^{2}$ film processed at $80^{\circ} \mathrm{C}$ was released within less than an hour. For the final analysis, however, the results from these incidents were rejected and the experiments (in threefold) were repeated to obtain sufficient data points to determine the average lateral etch rate of the here applied AlAs release layers as a function of temperature (see Fig. 3). The results confirm the predicted exponential behavior with temperature following from Eq. (2) and an activation energy, $E_{a}=0.22 \mathrm{eV}$ is deduced from the best linear fit through the data points in Fig. 3. Note that extrapolation of this linear relation also agrees well with the etch rate of a $5 \mathrm{~nm}$ $\mathrm{Al}_{0.85} \mathrm{Ga}_{0.15} \mathrm{As}$ layer at room temperature as deduced from Fig. 2.

As indicated by Figs. 2 and 3, both series of experiments show qualitative agreement with theory. Quantitative agreement is verified by replacement of $n$ by $n_{\max }$ which is the solubility of $\mathrm{H}_{2}$ in Eq. (1) and substitution of the constants by the appropriate values. Generally $R$ is in the order of $5 \mathrm{~cm}$ and $h$ was about $5 \mathrm{~nm}$ in most of the here described experiments. At room temperature $n_{\max } / N$ and $D$ were determined to be $\sim 2 \times 10^{-5}$ and $\sim 5 \times 10^{-5} \mathrm{~cm}^{2} / \mathrm{s}$, respectively, ${ }^{10,11}$ so that a maximum etch rate is derived of about $3 \mu \mathrm{m} / \mathrm{h}$. This value is several orders of magnitude less than the $3 \mathrm{~mm} / \mathrm{h}$ experimentally found in the present work and the recently reported values in literature (see Table I). Consequently, $\mathrm{H}_{2}$ gas cannot be a major reaction product if it is assumed that its transport from the etch front is correctly described by diffusion with a constant $D$ as stated above. Although etching of $\mathrm{AlGaAs}$ in $\mathrm{HF}$ has been reported to be a reaction-rate limited process, ${ }^{9}$ the value of $E_{a}=0.22 \mathrm{eV}$ as obtained in the present work is a strong indication that the process is diffusion rate limited as was also assumed by Yablonovitch et al. ${ }^{1}$ in order to derive Eq. (1). Based on the qualitative agreement 
TABLE I. Overview of applied curvature inducing mechanisms, typical process conditions, and resulting maximal lateral etch rates. RT stands for room temperature, $S 10$ indicates addition of a surfactant and antifoaming agent to the $10 \% \mathrm{HF}$ etch solution, and $p$ represents the pressure above the solution.

\begin{tabular}{crrlcc}
\hline \hline & $\begin{array}{c}h \\
\text { Curvature } \\
{[\mathrm{nm}]}\end{array}$ & $\begin{array}{c}T \\
{\left[{ }^{\circ} \mathrm{C}\right]}\end{array}$ & Other conditions & $\begin{array}{c}V_{e} \\
{[\mathrm{~mm} / \mathrm{h}]}\end{array}$ & Reference \\
\hline wax & $2-100$ & $\mathrm{RT}$ & standard configuration & 0.3 & 1,8 \\
wax & 50 & $\mathrm{RT}$ & $49 \%$ HF vapor phase & 0.125 & 8 \\
wax & 10 & 40 & $S 10$ & $\sim 1$ & 5 \\
screw & 10 & 50 & $S 10, p=5$ atm. & $\sim 2$ & 6 \\
weight & 3 & $\mathrm{RT}$ & & 3.0 & present work \\
weight & 5 & 80 & & 11.2 & present work \\
\hline \hline
\end{tabular}

but quantitative discrepancy between the here obtained results and established theory, we propose that $V_{e}$ is limited by out diffusion of a reaction product different from $\mathrm{H}_{2}$, e.g., a fluorine ligand with $\mathrm{Al}$ or As.

A high $V_{e}$ is considered essential for industrial applications. ${ }^{5,6}$ Therefore, recently $V_{e}$ as obtained by different approaches of the ELO method has been elaborated in literature (see Table I). From Table I it becomes clear that the WI-ELO technique at elevated temperatures has large potential provided that the released thin-film structures are of sufficient quality. In this respect it should be mentioned that, analogue to our previously reported GaAs thin films, ${ }^{2}$ none of the InGaP films reveal cracks. Hence, it was also possible to release and process several square centimeter sized InGaP thin-film structures to solar cells. The cell design was adapted from the structure reported by Takamoto et al. ${ }^{12}$ using a base thickness of $0.95 \mu \mathrm{m}$ and a back surface field doping level of $7 \times 10^{17} \mathrm{~cm}^{-3}$. Under standard solar illumination (AM1.5) we generally obtain efficiencies $(\eta)$ between $15 \%$ and $16 \%$ for such cells on GaAs substrates while a maximum of $\eta=18.5 \%$ has been reported in literature. ${ }^{12}$ Processing of thin-film InGaP cells took place by the socalled reversed sequence which is described in detail in previous work of our group on thin-film GaAs cells. ${ }^{2,3}$ The $J / V$ curve of the best cell obtained is characterized by a short circuit current density $J_{\mathrm{sc}}=11.0 \mathrm{~mA} / \mathrm{cm}^{2}$, an open circuit voltage $V_{\mathrm{oc}}=1.32 \mathrm{~V}$, and a fill factor $F F=0.79$ at a maximum power point of $9.97 \mathrm{~mA} / \mathrm{cm}^{2}$ and $1.15 \mathrm{~V}$. The resulting AM1.5 efficiency of $11.5 \%$ for this cell can be considered fair in comparison with the performance of its counter parts on a GaAs substrate for which we typically obtain $\eta$ $=15.5 \%\left(J_{\mathrm{sc}}=14.0 \mathrm{~mA} / \mathrm{cm}^{2}, V_{\mathrm{oc}}=1.35 \mathrm{~V}\right.$, and $\left.F F=0.82\right)$. The lower efficiency of the thin-film cells is probably a result of the additional difficulties encountered during handling and contacting these fragile structures.

The authors acknowledge financial support from the Dutch EET program and thank Jan van den Meerakker, Jan Weyher, and Ruud Tijburg for useful discussions with respect to III/V etching processes.

${ }^{1}$ E. Yablonovitch, T. Gmitter, J. P. Harbison, and R. Bhat, Appl. Phys. Lett. 51, 2222 (1987).

${ }^{2}$ P. R. Hageman, G. J. Bauhuis, A. van Geelen, P. C. van Rijsingen, J. J. Schermer, and L. J. Giling, Proceedings of the 25th IEEE Photovoltaic Specialists Conference (IEEE, New York, 1996), p. 57.

${ }^{3}$ A. van Geelen, P. R. Hageman, G. J. Bauhuis, P. C. van Rijsingen, P. Schmidt, and L. J. Giling, Mater. Sci. Eng., B 45, 162 (1997).

${ }^{4}$ Y. Yazada, J. Minemura, K. Tamura, S. Watahiki, T. Kitatani, and T. Warabisako, Sol. Energy Mater. Sol. Cells 50, 163 (1998).

${ }^{5}$ J. Maeda, Y. Sasaki, N. Dietz, K. Shibahara, S. Yokoyama, S. Miyazaki, and M. Hirose, Jpn. J. Appl. Phys., Part 1 36, 1554 (1997).

${ }^{6}$ Y. Sasaki, K. Katayama, T. Koishi, K. Shibahara, S. Yokoyama, S. Miyazaki, and M. Hirose, J. Electrochem. Soc. 146, 710 (1999).

${ }^{7}$ M. Konagai, M. Sugimoto, and K. Takahashi, J. Cryst. Growth 45, 277 (1978).

${ }^{8}$ W. Chang, C. P. Kao, G. A. Pike, J. A. Slone, and E. Yablonovitch, Sol. Energy Mater. Sol. Cells 58, 141 (1999).

${ }^{9}$ X. S. Wu, L. A. Coldren, and J. L. Merz, Electron. Lett. 21, 558 (1985).

${ }^{10}$ Treatise on Analytical Chemistry, Part II, Vol. 1, edited by I. M. Kolthoff, P. J. Elving, and E. B. Sandell (Interscience, New York, 1961).

${ }^{11}$ Kirk-Othmer Encyclopedia of Chemical Technology, Vol. 13, 4th ed. (Wiley, New York, 1995).

${ }^{12}$ T. Takamoto, E. Ikeda, H. Kurita, and M. Ohmori, Prodeedings of the 1st World Conference on Photovoltaic Solar Energy Conversion (IEEE, New York, 1994), p. 1729. 